\title{
The Evolution of Superconducting Phase $\mathrm{MgB}_{x}$
}

\author{
Sz. ŁoŚ ${ }^{a}$, W. Kempiński ${ }^{a}$, J. PieKOSZEWski ${ }^{b}$, \\ L. Piekara-SAdy ${ }^{a}$, Z. Werner ${ }^{b}$, M. BARlaK $^{b}$,
}

B. Andrzejewski ${ }^{a}$, W. Jurga ${ }^{a}$ And K. KASZYŃSKa ${ }^{a}$

${ }^{a}$ Institute of Molecular Physics, Polish Academy of Sciences

Smoluchowskiego 17, 61-179 Poznań, Poland

${ }^{b}$ The Andrzej Sołtan Institute for Nuclear Studies

05-400 Otwock/Świerk, Poland

Thin layers of $\mathrm{MgB}_{x}$ were studied in order to define evolution of superconducting phase after $\mathrm{Mg}$ ions implantation into boron substrate. Three fluencies of energies 140,80 , and $40 \mathrm{keV}$ were used to establish proper stoichiometry to synthesize homogeneous $\mathrm{MgB}_{2}$ film. Additionally, the annealing processes were carried out at temperatures 400,500 , and $600^{\circ} \mathrm{C}$ in a furnace in an atmosphere of flowing $\mathrm{Ar}-4 \% \mathrm{H}_{2}$ gas mixture. The quality of the superconducting material was examined by magnetically modulated microwave absorption, and magnetic and resistivity measurements. The results showed that $T_{\mathrm{c}}$ becomes higher with increasing annealing temperature. However, the fraction of superconducting phase decreases, due to partial evaporation of $\mathrm{Mg}$ ions and their deeper migration into boron substrate.

PACS numbers: 74.70.-b, 74.78.-w

\section{Introduction}

After discovery of superconductivity in $\mathrm{MgB}_{2}$ [1] much of an effort has been taken to obtain good quality thin film material with high $T_{\mathrm{c}}$ and high critical current $J_{\mathrm{c}}[2-4]$. This paper presents the continuation of our effort to synthesize thin layer of $\mathrm{MgB}_{2}$ with good superconducting properties by means of a boron ion implantation into magnesium and vice versa [5-8]. It has been shown that by changing the energy and the number of implanted ions as well as the post-implantation treatment it was possible to obtain samples with $T_{\mathrm{c}}$ 's close to crystalline value of $39 \mathrm{~K}$. One of the samples studied so far had the properties of superconducting which were characterized as having inhomogeneous island-like superconducting regions [9]. This paper shows how to improve the technology of ion implantation to obtain homogeneous thin layers of $\mathrm{MgB}_{2}$. 


\section{Experimental}

Three $\mathrm{MgB}_{x}$ samples were obtained by magnesium ion implantation into the bulk polycrystalline boron substrates. To get stoichiometry appropriate for $\mathrm{MgB}_{2}$, computer simulations using the stopping and range of ions in matter (SRIM) code were performed for multi-energy implantations. The computed fluencies were $6 \times 10^{17}, 3 \times 10^{17}$ and $3 \times 10^{17}$ ions $/ \mathrm{cm}^{2}$ for 140,80 , and $40 \mathrm{keV}$, respectively. Then, the samples were heated for 20 minutes at different temperatures, i.e. at 400,500 , and $600^{\circ} \mathrm{C}$ to remove radiation damage and stabilize the reaction in the solid state.

We have studied the quality of superconducting properties of the materials. First, Rutherford backscattering spectra (RBS) gave information about stoichiometry of $\mathrm{MgB}_{x}$ in the layer just below the surface. Three other methods, i.e. magnetically modulated microwave absorption (MMMA), magnetization and resistivity by four-point method tested superconducting properties of the samples.

\section{Results}

\subsection{Rutherford backscattering spectra}

RBS spectra show the presence of three separated peaks, not present in boron substrate (Fig. 1). Those are related to magnesium ions implanted on different depths. Comparison of the intensity of the spectrum for the sample annealed at $600^{\circ} \mathrm{C}$ with two others indicates that during heating at the highest temperature both, the content of magnesium is reduced and a part of ions diffuse deeper into the boron substrate.

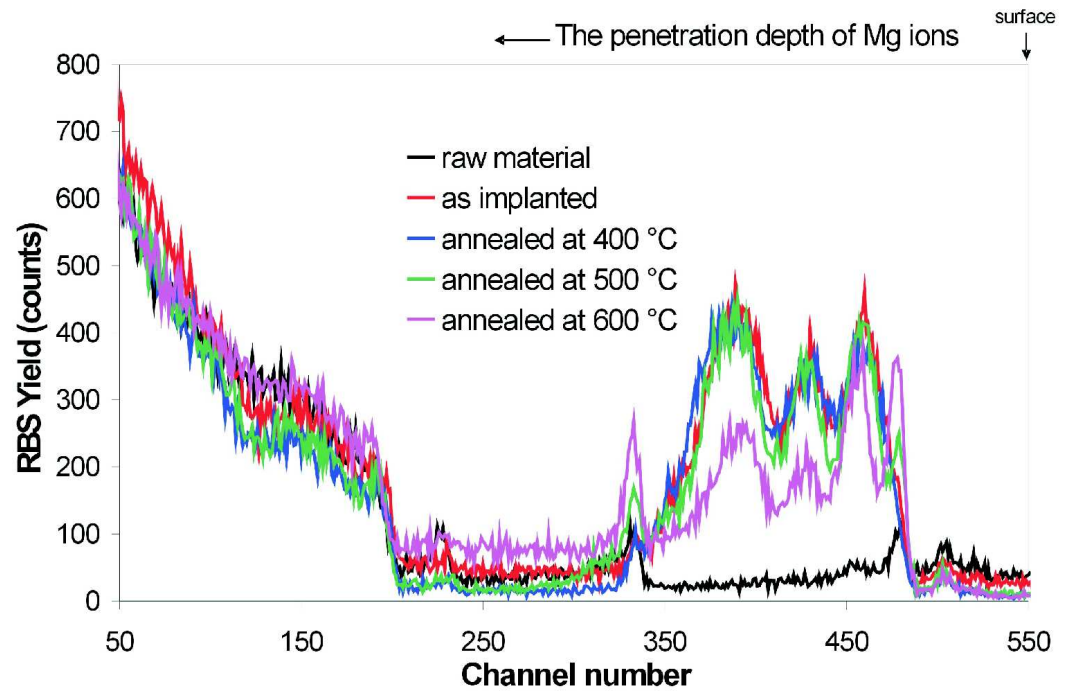

Fig. 1. Rutherford backscattering spectra recorded using $1.7 \mathrm{MeV} \mathrm{He}^{+}$ions beam at normal incident to the sample. 


\subsection{Magnetically modulated microwave absorption}

MMMA technique is a perfect method to determine temperature of the transition to the superconducting state, $T_{\mathrm{c}}$. Figure 2 presents $\operatorname{MMMA}(T)$ signals for

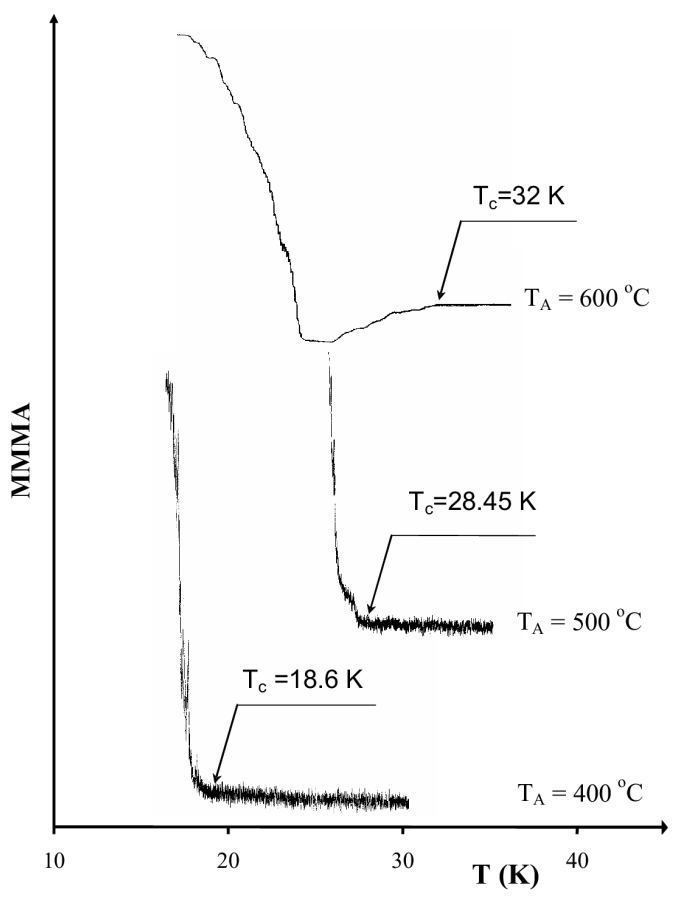

Fig. 2. The temperature dependences of MMMA for three different annealing temperatures.

the three samples. It is clearly seen that there is a strong correlation between the temperature of annealing $\left(T_{\mathrm{A}}\right)$ and $T_{\mathrm{c}}$. For $T_{\mathrm{A}}$ equal to 400,500 , and $600^{\circ} \mathrm{C}$ the values of $T_{\mathrm{c}}$ were about $18.6,28.5$, and $32 \mathrm{~K}$, respectively. Therefore, the highest annealing temperature resulted in better quality of the sample. The MMMA $(T)$ dependence shows also a specific feature - a negative signal just below $T_{\mathrm{c}}$. Such signal suggests that the sample contains isolated regions of superconducting phase.

\subsection{Resistivity measurements}

Temperature dependence of resistivity for the three samples supports the correlation between $T_{\mathrm{A}}$ and $T_{\mathrm{c}}$ observed by MMMA technique. Figure 3 shows $R(T)$ dependences. For $T_{\mathrm{A}}=400,500$, and $600^{\circ} \mathrm{C}$ the transition temperatures are: 20,28 , and $32 \mathrm{~K}$, respectively. However, none of the samples reached zero resistance and the transitions are wide. The width of the transition is the evidence that the superconducting phase exists in small regions of different quality, therefore their $T_{\mathrm{c}}$ 's are more or less different. The smallest $\Delta R$ (in \%) and largely diffused transition exhibit the sample heated at the highest temperature with the 


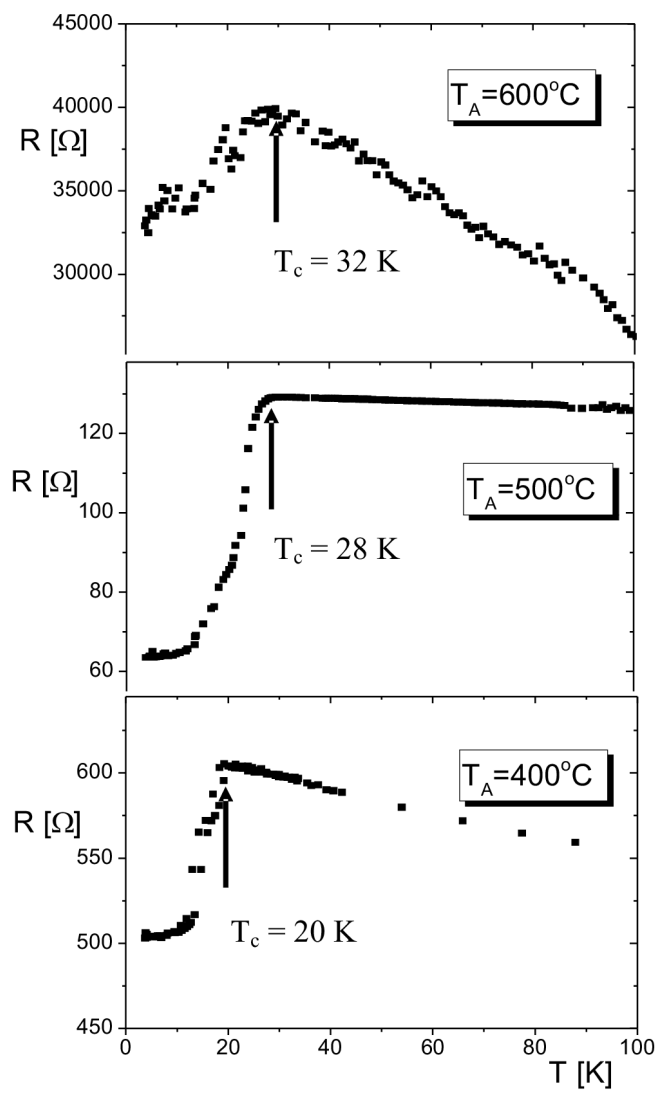

Fig. 3. Resistance temperature dependences for three different annealing temperatures.

highest $T_{\mathrm{c}}$. This means that there is a small amount of almost perfect $\mathrm{MgB}_{2}$ in this material.

\subsection{Magnetization measurements}

Magnetization measurements in $B=2 \mathrm{~T}$ supports the correlation of $T_{\mathrm{A}}$ with $T_{\mathrm{c}}$, i.e. the higher $T_{\mathrm{A}}$ the closer $T_{\mathrm{c}}$ to $39 \mathrm{~K}$, only for samples annealed at 400 and $500^{\circ} \mathrm{C}$. Figure 4 presents $M(T)$ dependences for $T_{\mathrm{A}}=400$ and $500^{\circ} \mathrm{C} . \Delta M$ for $T_{\mathrm{A}}=400^{\circ} \mathrm{C}$ is smaller than for the other temperature, which suggests that the superconducting volume fraction is smaller for lower temperature of the annealing. The width of the transition, $\Delta T$ is also smaller for the $400^{\circ} \mathrm{C}$ sample.

The third sample, $T_{\mathrm{A}}=600^{\circ} \mathrm{C}$, exhibited only increase in magnetic moment with decreasing temperature related to the iron impurity in boron substrate. 


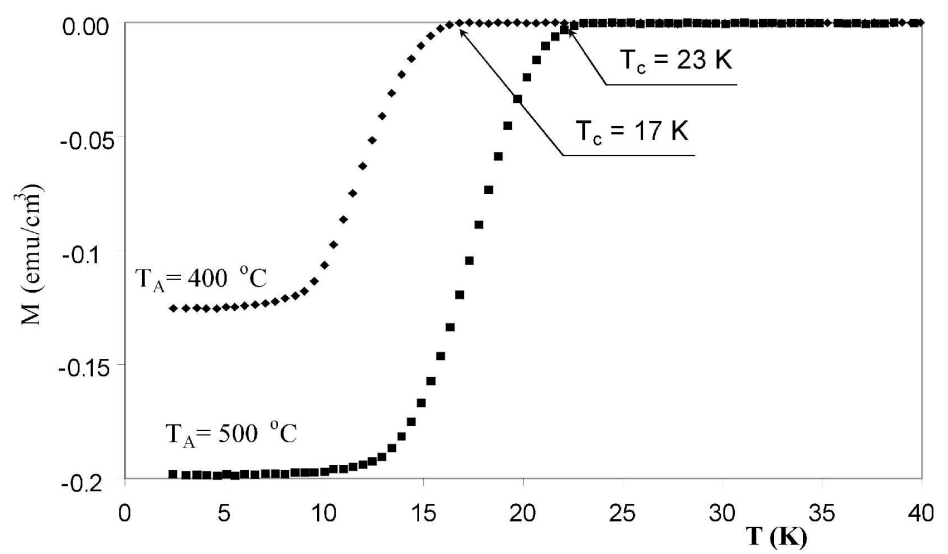

Fig. 4. Magnetization versus temperature for two different annealing temperatures.

\section{Discussion and conclusions}

The technology of ion implantation is a promising technique to obtain good quality inexpensive cheap thin layers of $\mathrm{MgB}_{2}$. However, the optimization of the parameters of this process is still needed.

RBS spectra showed that this technology is likely to produce a homogeneous layer of $\mathrm{MgB}_{2}$ of proper stoichiometry. Further process of annealing at 400 and $500^{\circ} \mathrm{C}$ resulted in the formation of superconducting regions with higher $T_{\mathrm{c}}$ for $500^{\circ} \mathrm{C}$. However, rising annealing temperature by another $100 \mathrm{deg}$ to $600^{\circ} \mathrm{C}$ caused the size reduction of the superconducting grains because of redistribution of magnesium ions by evaporation and diffusion deeper into the boron substrate. Due to their lower sensitivity, magnetization measurements showed no superconducting transition. Therefore, the magnetic field of $2 \mathrm{~T}$ penetrating the sample with small scattered grains of superconducting phase suggest the relation between the field penetration depth and the size of the grains. There is a profoundly diffused trace of the transition in resistivity measurements. For other samples the transition in resistivity implies an island-like character of superconducting material. $\Delta R$ amounts to $50 \%$ for $500^{\circ} \mathrm{C}$ sample. This is characteristic of substantial in size grains of superconducting phase.

The ion implantation technique followed by temperature treatment to obtain $\mathrm{MgB}_{2}$ thin films showed two competing effects:

1. increase in the temperature of annealing gives higher $T_{\mathrm{c}}$, very close to $39 \mathrm{~K}$

2. the highest temperature of annealing in these experiments resulted in formation of good quality superconducting phase $\left(T_{\mathrm{c}}=32 \mathrm{~K}\right)$, however, at the same time, the size of the grains of this phase is significantly diminished which is caused by magnesium redistribution and evaporation. 
The conclusion is obvious: the diffusion of implanted ions should be limited. A simple solution is to apply a pulse annealing. Results with e-beam annealing show that for the implanted samples e-beam annealing can activate the dopant fully with minimal redistribution while the furnace annealing substantially broaden the profile $[10]$.

\section{References}

[1] J. Nagamatsu, N. Nakagawa, T. Muranaka, Y. Zenitani, J. Akimitsu, Nature 410, 63 (2001).

[2] N. Peng, G. Shao, C. Jeynes, R.P. Webb, R.M. Gwilliam, G. Boudreault, D.M. Astill, W.Y. Liang, Appl. Phys. Lett. 82, 236 (2003).

[3] N. Peng, C. Jeynes, R.M. Gwilliam, K.J. Kirkby, R.P. Webb, G. Shao, D.M. Astill, W.Y. Liang, IEEE Trans. Appl. Supercond. 15, 3265 (2005).

[4] S. Soltanian, Ph.D. Thesis, University of Wollongong, Institute for Superconducting \& Mectronic Materials, Faculty Engineering, Australia 2004.

[5] J. Piekoszewski, W. Kempiński, J. Stankowski, F. Prokert, E. Richter, J. Stanisławski, Z. Werner, W. Szymczyk, Acta Phys. Pol. A 106, 861 (2004).

[6] J. Piekoszewski, W. Kempiński, B. Andrzejewski, Z. Trybuła, L. Piekara-Sady, J. Kaszyński, J. Stankowski, Z. Werner, E. Richter, F. Prokert, J. Stanisławski, M. Barlak, Vacuum 78, 123 (2005).

[7] Z. Trybuła, W. Kempiński, B. Andrzejewski, L. Piekara-Sady, J. Kaszyński, J. Piekoszewski, Z. Werner, E. Richter, F. Prokert, J. Stanisławski, Acta Phys. Pol. A 108, 165 (2005).

[8] J. Piekoszewski, W. Kempiński, M. Barlak, J. Kaszyński, J. Stanisławski, B. Andrzejewski, Z. Werner, L. Piekara-Sady, E. Richter, J. Stankowski, R. Grötzchel, Sz. Łoś, Vacuum 81, 1398 (2007).

[9] B. Andrzejewski, W. Kempiński, Z. Trybuła, J. Kaszyński, Sz. Łoś, J. Piekoszewski, J. Stanisławski, M. Barlak, J. Jagielski, R. Grötzschel, E. Richter, Cryogenics 47, 267 (2007).

[10] D.B. Rensch, J.Y. Chen, in: VLSI Science and Technology, Eds. C.J. Delloca, W.M. Bullis, Electrochemical Soc., Pennington 1982, p. 22. 